Commun. math. Phys. 39, 131-151 (1974)

(C) by Springer-Verlag 1974

\title{
Tilting at Cosmological Singularities
}

\author{
C. B. Collins \\ Department of Astrophysics, University of Oxford, Oxford, and Trinity College, Oxford, \\ England
}

Received January 19, 1974

\begin{abstract}
A detailed investigation is made of the simplest type of general relativistic perfect fluid cosmological models that possess a singularity at which all physical quantities are well-behaved. These models are spatially homogeneous, axisymmetric generalisations of the open $(k=-1)$ Robertson-Walker universes. A pictorial description of the evolution of the models is obtained by using the qualitative theory of differential equations.

The most surprising feature that emerges is that for some (non-empty) models the matter density may become zero, within a finite time, on a null hypersurface which acts as a Cauchy horizon for the models. This result is generalized to most other types of spatially homogeneous models.

It is also discovered that the behaviour of the models varies dramatically with the type of matter content. This casts some doubts on the validity of assuming definite equations of state in general relativity, and suggests an investigation of the structural stability of Einstein's field equations.
\end{abstract}

\section{Introduction}

Ever since the discovery that the spatially homogeneous and isotropic Robertson-Walker models of the universe possess a "big-bang" singularity, at which the density of matter and the curvature of space-time become infinite, conventional cosmology has seized the opportunity to interpret it as a theoretical triumph heralding the birth of our universe. Moreover, any suspicions that the existence of the singularity is a consequence entirely of the very high symmetry of the models have since proved to be unfounded. Powerful theorems, established chiefly by Hawking and Penrose ([1], and references cited therein), require that, under certain reasonable conditions, any sufficiently general solution of Einstein's field equations of general relativity will possess a singularity; subsequent work of Ellis, Sciama, and Hawking $[2,3]$ shows that our universe will indeed satisfy the requirements for the theorems to hold.

However, the type of singularity that the Hawking-Penrose theorems entail is more general than the big-bang sort. Rather more subtle concepts, relating to the inextendibility of causal (timelike or null) curves in the space-time manifold have had to be introduced. 
Contemporaneously with the development of these theorems, cosmologists were generalising the Robertson-Walker models in an effort to study the behaviour of more realistic universe models. For reasons of both physical plausibility and mathematical tractability, the assumption of spatial homogeneity was retained, while that of isotropy was relaxed. This led from a suggestion of Gödel [4], through the pioneering works of Taub [5] and Heckmann and Schücking [6], to the establishment of a classification scheme for the ten (Bianchi) types of spatially homogeneous anisotropic cosmological models [7], and to a subsequent extensive investigation of each type [6-10].

The singularity that occurred in these anisotropic models was generally either proved or tacitly assumed to be of the big-bang type, and even when Shepley [11] pointed out that some other sort was possible, cosmologists still clung to the viewpoint that such examples were far-fetched and did not typify any general state of affairs. Whilst this may turn out to be correct, the reasons for believing it to be the case were based to some extent on wishful thinking and dangerous extrapolation, and a rigorous investigation of the nature of the singularity in spatially homogeneous anisotropic models has commenced only recently. Ellis and King [12] show that, in a moderately large subclass of these models, there is a "breakdown of prediction", caused by the hypersurfaces of homogeneity, $S$, changing their spatial character, so that the worldlines of the matter content can pass, within a finite time, from a spatially homogeneous region into an inhomogeneous but stationary region ${ }^{1}$. The interface between these two regions of spacetime is a Cauchy horizon ([15], called colloquially a "whimper" in [12]), $H^{+}(S)$. All physical quantities are well-behaved on $H^{+}(S)$, but nevertheless the models are singular in a well-defined sense. The type of singularity that arises is naturally free of difficulties relating to the physics of elementary particles at high energies, such as are prevalent in the simple big-bang singularities. The detailed examination of what may happen beyond $H^{+}(S)$, and of the possible combinations of whimpers and singular beginnings and ends of different universe models, forms the subject matter of Ellis and King's paper.

The restriction to spatial homogeneity is made by cosmologists with some degree of resignation. One would naturally like to know, for instance, the nature of the singularity in general inhomogeneous universe models, but this question appears to be extremely difficult, having eluded all rigorous attempts to come to grips with it. The advantage of homogeneous models rests primarily on the study of ordinary (as

1 Taub-NUT space is a vacuum solution which has similar properties. It consists of a spatially homogeneous region, investigated by Taub [5], which separates two stationary inhomogeneous regions considered by Newman, Tamburino, and Unti [13]. Further details are given in the work of Misner and Taub [14]. 
opposed to partial) differential equations; even this can involve a fairly complicated analysis owing to the highly nonlinear nature of the field equations. Computations are facilitated whenever a Lagrangian formalism can be introduced (cf. $[8,16]$ and references cited therein), and a potential wall description can then give a vivid intuitive picture of the behaviour of the solutions. However, this method is not always reliable, and a more precise procedure (based for instance on the qualitative theory of differential equations) can reveal its inadequacies [17]. One would anticipate that the detailed examination of the whimper sort of singularity would involve fairly delicate arguments, for which a Lagrangian formalism (even if it existed) could not be employed, and in that case an alternative method would have to be sought. Ellis and King use a combination of topological arguments and a detailed analysis of the field equations, employing differential inequalities. The qualitative theory of differential equations would provide a second possibility, but it suffers from the disadvantage that it can be employed only for sufficiently simple systems. However, there is one case where this theory can be used successfully to analyse in extensive detail the behaviour of the simplest type of model that admits whimpers: an axisymmetric (i.e., locally rotationally symmetric, or LRS) Bianchi type V model containing a perfect fluid with equation of state $p=(\gamma-1) \mu$, where $\gamma$ is a constant, $p$ is the isotropic pressure, and $\mu$ is the total energy density. This model, which generalises the open $(k=-1)$ Robertson-Walker model, has been considered in the case of dust (incoherent matter; $p=0$ ) by Farnsworth [18], Shepley [11], and Shikin [19], and for more general matter content by Stewart, King, and Ellis [10,20].

It is the purpose of the present paper to investigate further these models, particularly with a view to analysing their asymptotic behaviours (big-bangs, whimpers, etc.) and to extending the models analytically beyond their Cauchy horizons. In Section 2, the necessary formalism is set up. Some brief remarks are made in Section 3 on how the qualitative study is performed, and in Section 4 the behaviour of models is described for various values of $\gamma$ [the most physically relevant values being 1 (dust), $4 / 3$ (radiation) and 2 (Zeldovich stiff matter)]. Stationary inhomogeneous counterparts are discussed in some detail. Certain results obtained in this way are generalised to more complicated models (e.g. to other Bianchi types) in Section 5, and Section 6 concludes with some remarks which in particular highlight the results of Section 4.

\section{The Models: Formalism}

(i) Spatially Homogeneous Region. A spatially homogeneous model of the universe is described in terms of a continuous group of isometries which acts transitively on a family of spatial hypersurfaces, $S(\tilde{t})$, param- 
etrized by a time variable, $\tilde{t}$. One can then employ an orthonormal tetrad technique ([8-10], and references cited therein) to analyse the field equations. Using a "normal basis" $\left\{\boldsymbol{n}, \tilde{\boldsymbol{e}}_{\mu}\right\}$ in which $\boldsymbol{n}$ is the geodesic normal to $S(\tilde{t})$ and $\tilde{\boldsymbol{e}}_{\mu}$ (Greek indices take values 1,2 , and 3, and Latin indices take values $0,1,2,3)$ is a basis of vectors which spans $S(\tilde{t})$ and is invariant under the group, we form the commutation relations ${ }^{2}$

$$
\left[\tilde{\boldsymbol{e}}_{\kappa}, \tilde{\boldsymbol{e}}_{\mu}\right]=\left(\varepsilon_{\kappa \mu \tau} h^{\tau \nu}+\delta_{\mu}^{v} a_{\kappa}-\delta_{\kappa}^{v} a_{\mu}\right) \tilde{\boldsymbol{e}}_{v}
$$

where $n^{\mu v}=n^{\mu v}(\tilde{t})=n^{(\mu v)}, a_{\kappa}=a_{\kappa}(\tilde{t})$ and $n^{\alpha \beta} a_{\beta}=0$.

This last requirement forms the foundation for a group classification scheme, and when $n^{\alpha \beta}=0$ and $a_{\beta} \neq 0$ the group is said to be of Bianchi type $V$. A space-time is locally rotationally symmetric (LRS) if at each point there is a group of rotations about a spacelike axis, under which all covariantly defined quantities are invariant [20]. In the particular case of a LRS model that is spatially homogeneous of Bianchi type $V$, a coordinate system can be chosen $[10,18]$ so that the metric is of the form

$$
d s^{2}=-d \tilde{t}^{2}+X^{2}(\tilde{t}) d x^{2}+Y^{2}(\tilde{t}) e^{-2 x}\left(d y^{2}+d z^{2}\right) .
$$

The matter will not in general move along the normals, $\boldsymbol{n}$, to $S(\tilde{t})$, and its flow vector $\boldsymbol{u}$ is conveniently described using a hyperbolic angle of tilt, $\psi \geqq 0$, where $\cosh \psi:=-\boldsymbol{u} \cdot \boldsymbol{n}, \boldsymbol{u} \cdot \boldsymbol{u}=-1$ and $\boldsymbol{n} \cdot \boldsymbol{n}=-1$; thus $\psi=0 \Leftrightarrow \boldsymbol{u}=\boldsymbol{n}$. There are three pairs of equations:

(i) the conservation equations

and

$$
\left(\ln w X Y^{2} \cosh \psi\right)^{\circ}=\frac{2}{X} \tanh \psi
$$

$$
(\ln r \sinh \psi)^{\circ}=-\frac{\dot{X}}{X}
$$

(ii) the field equations

and

$$
\frac{\ddot{X}}{X}+\frac{2 \dot{X}}{X} \frac{\dot{Y}}{Y}-\frac{2}{X^{2}}=\frac{1}{2}(\mu-p)+(\mu+p) \sinh ^{2} \psi,
$$

$$
\frac{\ddot{Y}}{Y}+\frac{\dot{Y}^{2}}{Y^{2}}+\frac{\dot{X}}{X} \frac{\dot{Y}}{Y}-\frac{2}{X^{2}}=\frac{1}{2}(\mu-p),
$$

and (iii) the first integrals

$$
\frac{2}{X}\left(\frac{\dot{X}}{X}-\frac{\dot{Y}}{Y}\right)=(\mu+p) \sinh \psi \cosh \psi
$$

${ }^{2}$ Given two vector fields, $\boldsymbol{X}$ and $\boldsymbol{Y}$, the commutator $[\boldsymbol{X}, \boldsymbol{Y}]$ is the vector field with components $[\boldsymbol{X}, \boldsymbol{Y}]^{i}=Y_{; j}^{i} X^{J}-X^{i}{ }_{j j} Y^{j}$; it is also characterized as the Lie derivative of $\boldsymbol{Y}$ along $\boldsymbol{X}$. 
and

$$
\frac{2 \dot{X}}{X} \frac{\dot{Y}}{Y}+\frac{\dot{Y}^{2}}{Y^{2}}=\frac{3}{X^{2}}+\mu \cosh ^{2} \psi+p \sinh ^{2} \psi
$$

In these equations, a $\operatorname{dot}\left({ }^{\circ}\right)$ denotes differentiation with respect to $\tilde{t}$, the quantities $w>0$ and $r>0$ are defined for $\mu>0$ by $\frac{d w}{w}:=\frac{d \mu}{\mu+p}$, $\frac{d r}{r}:=\frac{d p}{d \mu} \cdot \frac{d \mu}{\mu+p}$, and we have taken the cosmological constant to be zero. If we now make the substitutions

$$
X:=e^{-\Omega+\beta}, \quad Y:=e^{-\Omega-\beta / 2}, \quad x:=\frac{1}{3} \frac{\mu}{\dot{\Omega}^{2}}, \quad A:=\frac{1}{X \dot{\Omega}}
$$

and $p=(\gamma-1) \mu$, where $\gamma$ is a constant satisfying $1 \leqq \gamma \leqq 2$, we can rewrite Eqs. (2.2)-(2.5) in terms of two new dependent variables $\beta$ and $v:=\tanh \psi$, and an independent variable $\Omega$ (cf. $[8,20]$ ); derivatives with respect to $\Omega$ will be denoted by a prime ('). The quantity $e^{-\Omega}$ measures average length scales in the hypersurface $S(\tilde{t}), \beta$ is a measure of the degree of distortion of the metric from isotropy, $\beta^{\prime}$ measures the dynamical importance of the shear of the normals to $S(\tilde{t}), x$ measures the importance of matter, and $v$ is the magnitude of the peculiar velocity of the matter relative to $S(\tilde{t})$. Upon eliminating the matter terms, Eq. (2.2) yield a single equation for $\psi$ :

$$
\psi^{\prime}=-\frac{\left[\left(3 \gamma-4+\beta^{\prime}\right) s c+2 A(\gamma-1) s^{2}\right]}{1+(2-\gamma) s^{2}}
$$

where $s:=\sinh \psi$ and $c:=\cosh \psi$. Similarly, by eliminating $\ddot{\Omega}$ terms from Eq. (2.3), they yield

$$
\beta^{\prime \prime}=2 A^{2} \beta^{\prime}+\frac{3}{2}(2-\gamma) x \beta^{\prime}+\gamma x\left(2+\beta^{\prime}\right) s^{2}
$$

whilst Eqs. (2.4) and (2.5) become

and

$$
\begin{gathered}
\gamma_{x s c}=A \beta^{\prime} \\
4=\beta^{\prime 2}+4 x\left(1+\gamma s^{2}\right)+4 A^{2} .
\end{gathered}
$$

By eliminating $A$ from $\left(2.4^{\prime}\right)$ and $\left(2.5^{\prime}\right)$ we arrive at a quadratic equation for the variable $x$, whose solutions for $\psi \neq 0$ are

$$
x=\frac{-\beta^{\prime 2}\left(1+\gamma s^{2}\right) \pm\left[\beta^{\prime 4}\left(1+\gamma s^{2}\right)^{2}+\gamma^{2} s^{2} c^{2}\left(4-\beta^{\prime 2}\right)\right]^{1 / 2}}{2 \gamma^{2} s^{2} c^{2}} .
$$

In the spatially homogeneous region, $x=\frac{1}{3} \frac{\mu}{\dot{\Omega}^{2}} \geqq 0$, and so we must choose the positive root in Eq. (2.6). Using Eqs. $\left(2.2^{\prime}\right)-\left(2.4^{\prime}\right)$ and (2.6) 
we finally arrive at a pair of autonomous propagation equations for $v$ and $\beta^{\prime}$ :

$v^{\prime}=-\frac{\gamma\left(3 \gamma-4+\beta^{\prime}\right) v^{2}+(\gamma-1)\left[-\beta^{\prime}\left(1+(\gamma-1) v^{2}\right)+\left(\operatorname{sgn} \beta^{\prime}\right) \Delta^{1 / 2}\right]}{\gamma v\left[1-v^{2}(\gamma-1)\right]}$

$$
\begin{aligned}
\beta^{\prime \prime}= & \frac{\beta^{\prime}}{4 \gamma^{2} v^{2}}\left\{2 \gamma^{2}\left(4-\beta^{\prime 2}\right) v^{2}+\left[2 \gamma\left(2-\beta^{\prime}\right) v^{2}+\beta^{\prime}(3 \gamma-2)\left(1-v^{2}\right)\right]\right. \\
& \left.\cdot\left[-\beta^{\prime}\left(1+(\gamma-1) v^{2}\right)+\left(\operatorname{sgn} \beta^{\prime}\right) \Delta^{1 / 2}\right]\right\}
\end{aligned}
$$

where $\Delta:=\beta^{\prime 2}\left[1+(\gamma-1) v^{2}\right]^{2}+\gamma^{2} v^{2}\left(4-\beta^{\prime 2}\right)$. The expressions for $x$, $A, v^{\prime}$, and $\beta^{\prime \prime}$ in Eqs. (2.6)-(2.8) for $\psi=0$ can be obtained from continuity requirements (i.e. by letting $\psi \rightarrow 0$ ), except in the case where $\beta^{\prime} \rightarrow 0$ also, when the square root term gives rise to a directional limit. Note that Eq. (2.7) assumes a particularly simple form for the case $\gamma=1$ (the Farnworth dust solution).

(ii) Stationary Inhomogeneous Region. We examine stationary inhomogeneous models of Bianchi type $V$ by generalising our requirement of Section 2 (i) that $\psi$ be real. In those models which possess whimpers, the hypersurfaces of homogeneity change their spacelike character and become timelike. This means that values of $v=\tanh \psi$ exceed unity, and so $\psi$ becomes imaginary, being of the form

$$
\psi=\phi+\frac{i \pi}{2}
$$

where $\phi$ is real. Then $\sinh \psi=i \cosh \phi$ and $\cosh \psi=i \sinh \phi$. We can therefore determine how the formalism of King and Ellis [10] and of Section 2 (i) above will change on substituting Eq. (2.9) throughout. The term $X^{2}(\tilde{t})$ in the metric (2.1) changes sign; thus $X$ becomes purely imaginary, whilst $Y(\tilde{t})$ remains real. This means that for $v>1, \frac{d \beta}{d \Omega}$ and $\frac{d \psi}{d \Omega}=\frac{d \phi}{d \Omega}$ remain real. Since $d \tilde{t}^{2}$ changes sign, $\dot{\Omega}^{2}$ becomes negative, and therefore $x=\frac{1}{3} \frac{\mu}{\dot{\Omega}^{2}}<0$. The negative root in Eq. (2.6) is now appropriate; this implies that $\beta^{2} \leqq 4$. Equations (2.7) and (2.8) maintain their validity. They hold also for stationary space-times, in which $v$ always exceeds unity.

Note that for $v>1$ and $\gamma \neq 1$ it is possible for the term $1-v^{2}(\gamma-1)$ in the denominator of (2.7) to vanish. We shall see in Section 4 that this gives rise to a variety of possible behaviours in the stationary region, but that none of these is analogous to the behaviour exhibited by the dust model $(\gamma=1)$ in which this denominator term cannot vanish and a matter singularity is encountered within a finite time. 
Note also that in general the extension of a universe model across its Cauchy horizon $H^{+}(S)$, is not unique. Uniqueness can be achieved in the perfect fluid case by assuming, for instance, that the continuation across $\mathrm{H}^{+}(S)$ is analytic, which is equivalent to demanding that the continuation admit locally a continuous 3-parameters isometry group acting transitively on timelike hypersurfaces ([12]; cf. the vacuum Taub-NUT space, which possesses at least two distinct analytic extensions [14]).

\section{The Qualitative Method}

The qualitative theory of ordinary differential equations yields a pictorial description of solutions of a pair of coupled non-linear ordinary differential equations of the form

$$
\begin{aligned}
& \dot{x}=X(x, y) \\
& \dot{y}=Y(x, y),
\end{aligned}
$$

a "plane autonomous system", where a dot (') denotes differentiation with respect to some extraneous variable. In general relativistic cosmology, this theory has been utilized by the present author and by Shikin [17] to obtain diagrams which depict the evolution of a class of spatially homogeneous anisotropic models in which the matter flows orthogonally to the hypersurfaces of homogeneity (i.e., $\psi \equiv 0$ ). A set of "streamlines" is obtained, each one representing the evolution of a model (or a subset of models), and information relating to such questions as initial and final states can be immediately determined.

The solutions of Eq. (3.1) are visualized as continuous curves in the $x-y$ phase-space, and can be interpreted as the streamlines of a fluid particle moving in two dimensions. In order to obtain a global theory, it is assumed that phase-space does not have the topology of the torus, $\mathscr{S} \times \mathscr{S}$, i.e., that the dependent variables do not both behave as angles (with this assumption, any streamline provides a barrier for any other: a closed curve necessarily divides the plane, $\mathscr{R} \times \mathscr{R}$, or the cylinder, $\mathscr{R} \times \mathscr{S}$, into two parts, whereas this need not happen on a torus. Phasespace is necessarily formed as the Cartesian product of two variables). In the cases that we shall be considering, this topological condition will be satisfied.

The first step is to determine the local behaviour, i.e., to examine the behaviour of the streamlines in the neighbourhood of "singular points" [these are points $(x, y)$ for which $X \equiv Y \equiv 0$ in (3.1)]. It is usually required that these singular points be isolated, although this is not always essential [cf. Section 4 (v)]. We consider the linearized equations 
(in the neighbourhood of each singular point). These can be solved exactly, or put into a canonical form and classified. It is then shown that under certain circumstances the inclusion of the non-linear terms does not cause any qualitative change in the local behaviour of the solutions. Further information can be gained by considering the "isoclines" (for which either $X \equiv 0$ or $Y \equiv 0)$; details may be found in the standard books [21].

If in addition to the local behaviour we can determine the behaviour of streamlines at infinity (whenever this is relevant), then in order to obtain a completely global picture we need only join up local to distant portions. This procedure can often be carried out in a unique fashion, but one major obstacle that remains is the possible existence of "limit cycles", or closed streamlines. Considerable understanding has been achieved towards the resolution of the question of limit cycles, several important results being due to, or arising from, the work of Bendixson [22]. Details of these results can be obtained in the standard works on the subject [21].

In the next section we carry out the qualitative analysis of the plane autonomous systems (2.7) and (2.8). Instead of using the quantity $v$ as the tilt-variable, it will be more convenient to consider $V:=2\left(1+v^{2}\right)^{-1}$, which assumes values in the interval $[0,2]$ and thereby confines our diagrams to a finite region of the plane. Each figure will consist of a part $(1<V \leqq 2)$ representing spatially homogeneous regions of spacetime, and a part $(0 \leqq V<1)$ representing stationary inhomogeneous regions. The interface $(V=1)$ will depict the Cauchy horizon (whenever this exists). In these diagrams (see Figs. $1-5$ ), each streamline will describe the evolution, either in time $(1<V \leqq 2)$ or in space $(0 \leqq V<1)$, of a family of models; consequently each figure depicts simultaneously the behaviour of an entire class of solutions. These diagrams contrast, therefore, with the conformal space-time diagrams (cf. $[12,15])$ for the models, in which each point represents a 2 -surface diffeomorphic to $\mathscr{R} \times \mathscr{R}$; consequently each conformal diagram represents the behaviour of any one member of a class of solutions.

\section{Tilted LRS Models of Type V}

We now examine the different behaviours of tilted models $(\psi \neq 0$ or $\phi \neq 0$ ) which are invariant under a 4-parameter isometry group acting multiply transitively on hypersurfaces $S(\tilde{t})$ and which contain a perfect fluid $^{3}$ with equation of state $p=(\gamma-1) \mu$. It is convenient to consider

${ }^{3}$ King and Ellis have shown that the only tilted spatially homogeneous LRS perfect fluid models are of type $V$ [10]. 
five qualitatively distinct types of behaviour, according as (i) $\gamma=1$, (ii) $1<\gamma<4 / 3$, (iii) $\gamma=4 / 3$, (iv) $4 / 3<\gamma<2$ and (v) $\gamma=2$. The results are tabulated in Table 1, in which for completeness have been included the Robertson-Walker models. From Eq. $\left(2.4^{\prime}\right)$ we see that these are the only non-tilted models ( $\psi \equiv 0$ or $\phi \equiv 0 \Rightarrow \beta^{\prime} \equiv 0$ and $\psi \equiv 0$ ).

(i) $\gamma=1$. In this case the matter consists of dust $(p=0)$. This model was first examined by Farnsworth [18] and studied further by Ellis [20] and Shikin [19]. There are three types of tilted model (see Fig. 1):

(A) The simplest type is one for which the entire manifold is stationary and inhomogeneous and in which there are two timelike matter singularities, both attained within a finite time. The expansion and shear of the matter congruence become infinite, but similar quantities relating to the normal congruence remain well-behaved (see Table 1 for details). The singularity is of the pancake type (see Thorne [23] for a description of matter singularity types in terms of "pancakes", "barrels", "points", and "cigars").

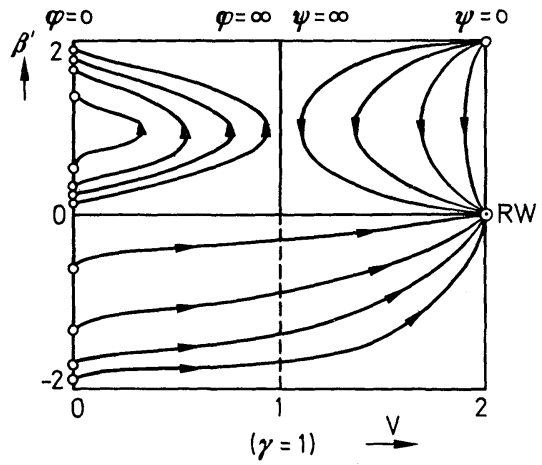

Fig. 1

Figures 1-5 depict the evolution of perfect fluid LRS Bianchi type $V$ models, in terms of the variables $\beta^{\prime}$ (which is a measure of the dynamical importance of shear anisotropy) and $V:=2\left(1+\tanh ^{2} \psi\right)^{-1}$, where $\psi$ is the "hyperbolic angle of tilt" which measures the inclination between the 4-velocity, $u^{a}$, of the matter and the normals, $n^{a}$, of the homogeneous hypersurfaces according to $\cosh \psi=-u^{a} n_{a}$. Values of $V$ in the range $1<V \leqq 2$ $(\psi \geqq 0)$ correspond to spatially homogeneous regions, and values in the range $0 \leqq V<1\left(\psi=\phi+\frac{i \pi}{2}, \phi \geqq 0\right)$ correspond to stationary inhomogeneous regions, whilst $V=1$ corresponds to the interface between the two (i.e. a Cauchy horizon). Disjoint parts, consisting of a subset of models with the same qualitative behaviour, are bounded by thick lines, and small circles denote the initial and final states of the evolution of the models. Arrows are drawn so that in the spatially homogeneous part the models are expanding either from an initial state of zero volume or towards a final state of zero density. The isotropic Robertson-Walker models which have zero tilt and shear, lie at the point $(2,0)$ and are denoted by $R W$ 

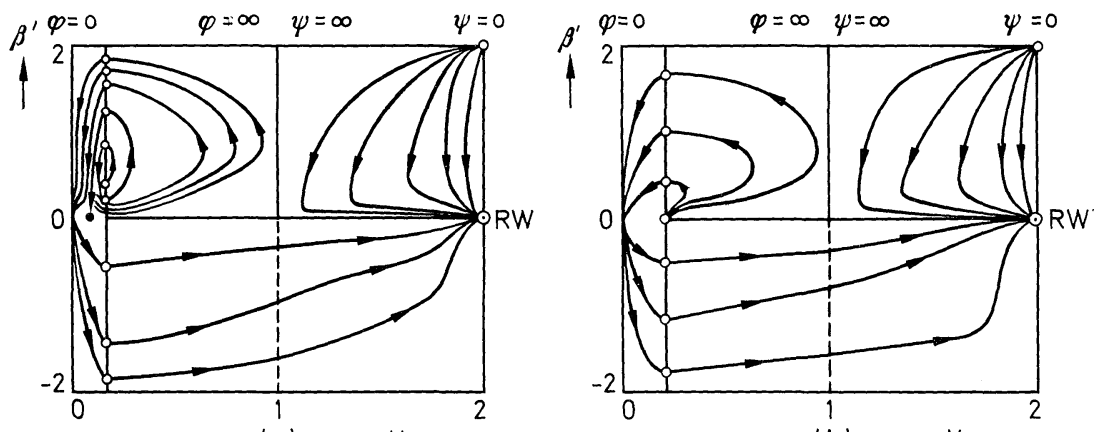

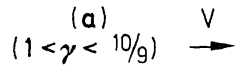

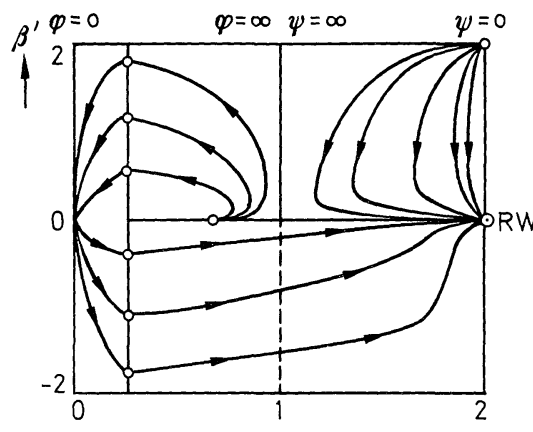

$(c)$
$(10 / 9<\gamma<6 / 5)$

$(1<\gamma<4 / 3)$

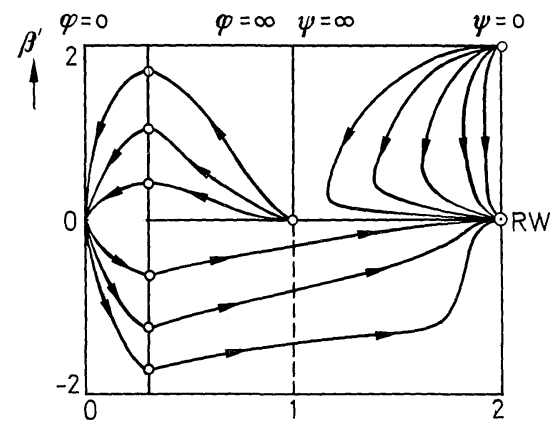

(d)

$6 / 5 \leq \gamma<4 / 3) \rightarrow$

Fig. 2

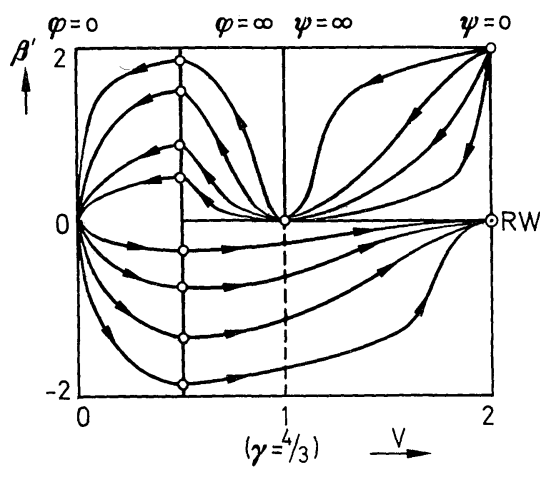

Fig. 3

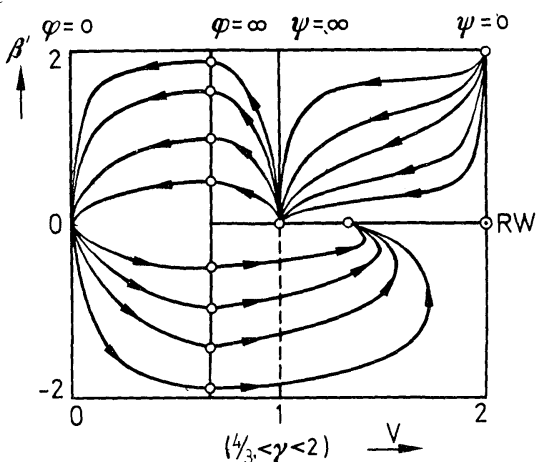

Fig. 4

(B) The next simplest type of solution is one representing universe models in which the matter expands out of a highly anisotropic (cigar) infinite-density singularity (a big-bang). The fluid shear and expansion become infinite although the curvature scalar of the homogeneous 


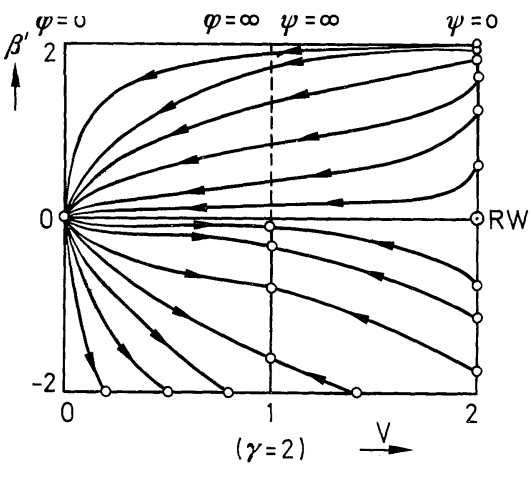

Fig. 5

hypersurfaces tends to zero. At large times the models approach isotropy $\left(\beta^{\prime} \rightarrow 0, \psi \rightarrow 0\right)$ and the matter density becomes negligible (cf. [9]).

(C) The third type of solution possesses a Cauchy horizon, $\mathrm{H}^{+}(S)$. It was exhibited by Shepley [11] as an example of a solution to the Einstein field equations in which the existence of a singularity does not necessitate an infinite matter density. It is the simplest model with this property; further details can be found in [12]. In the spatially homogeneous region the homogeneous hypersurfaces tilt so much that they become null within a finite proper time; as the flowlines pass through $\mathrm{H}^{+}(S)$, all physical quantities connected with the matter flow remain well-behaved. Analytic continuation through $\mathrm{H}^{+}(S)$ shows that, in the stationary region, a pancake matter singularity is encountered within a finite proper time. We see from Fig. 1 and Table 1 that at large times these models approach isotropy $\left(\beta^{\prime} \rightarrow 0, \psi \rightarrow 0\right)$ in the spatially homogeneous region [9].

(ii) $1<\gamma<4 / 3$. There is now a great variety of possibilities in the inhomogeneous region, as depicted in Fig. $2 \mathrm{a}-\mathrm{d}$, for $1<\gamma<10 / 9$, $\gamma=10 / 9,10 / 9<\gamma<6 / 5$, and $6 / 5 \leqq \gamma<4 / 3$ respectively ${ }^{4}$.

(A) A large proportion of stationary inhomogeneous models possess two singularities, which are encountered along the flowlines of the matter within a finite proper time. The fluid shear, expansion and acceleration become infinite, although the matter density remains finite. The singularity is therefore of the "conformal curvature" type (cf. [12]). This is the only sort of stationary inhomogeneous model for values of $\gamma$ satisfying $1<\gamma<10 / 9$.

(B) The remaining inhomogeneous solutions $(10 / 9 \leqq \gamma<4 / 3)$ possess only one conformal curvature singularity. The worldlines of the fluid can be extended away from this singularity for infinitely large values of

4 In drawing Fig. 2a we have assumed that no limit cycles (closed stream-lines) occur. The existence of limit cycles would not change the nature of the solutions. 
proper time, $t$, the complex angle of tilt becoming infinite for values of $\gamma$ in the range $6 / 5 \leqq \gamma<4 / 3$.

(C) As in Section 4 (i) above, there are manifolds which are spatially homogeneous throughout. These start at a highly anisotropic cigar singularity (at which the fluid density, $\mu$, expansion, $\theta$, shear, $\sigma$, and acceleration, $\dot{u}$, are all infinite, but the curvature scalar, $R^{*}$ of $S(\tilde{t})$ is zero) and at large times they approach isotropy $\left(\beta^{\prime} \rightarrow 0, \psi \rightarrow 0\right)$.

(D) The final type of model is one which admits a Cauchy horizon. The properties are similar to those of the dust case, except that in the inhomogeneous region a conformal curvature singularity is encountered $\left(\theta, \sigma\right.$, and $\dot{u}$ infinite yet $\mu$ and $R^{*}$ are finite and non zero) within a finite time.

(iii) $\gamma=4 / 3$. The matter now consists of an ultrarelativistic gas, which could be the appropriate content for a model of the early stages of the universe. The various possible cases (see Fig. 3) are similar to Case (ii) above, except for the late stages of the spatially homogeneous models. The difference in this case is that the angle of tilt, $\psi$, becomes infinite. Thus although the models do approach isotropy in some sense $(\tilde{\sigma} / \tilde{\theta} \rightarrow 0$, where $\tilde{\theta}$ and $\tilde{\sigma}$ are respectively the expansion and shear of the normals), they do not in others: $\psi \rightarrow \infty$ and the fluid shear, expansion and acceleration approach finite but non-zero limits (cf. [9]).

(iv) $4 / 3<\gamma<2$. These models are similar to the radiation Case (iii) above, but the tilt of these models never tends to zero in the spatially homogeneous region. Thus provided that the matter is hard enough, it will prevent the models from approaching isotropy in the sense of [9]. Moreover, the spatially homogeneous models do not last indefinitely, but expand out to infinite proper volume within a finite proper time. Details are shown in Fig. 4.

(v) $\gamma=2$. In this limiting case, there are three types of solution (see Fig. 5):

(A) These are the stationary inhomogeneous models in which the world-lines of the fluid can be extended for infinitely large proper times in both directions. In one direction, $\mu, \sigma$, and $\dot{u}$ remain finite and non-zero, $\theta \rightarrow 0$ and yet length-scales remain finite. This situation is achieved within a finite "normal" time, $\tilde{t}$. In the opposite direction, $\mu, \theta, \sigma$, and $\dot{u}$ all tend to zero and length-scales become infinite.

(B) Some solution give rise to manifolds which are spatially homogeneous throughout. These start at a matter singularity at which $\mu, \theta$, $\sigma$, and $\dot{u}$ are all infinite. If the limiting value of $\beta^{\prime}$ is greater than -2 , one obtains a point singularity (length-scales along all eigendirections of the shear tensor $\sigma_{a b}$ tend to zero), however, in the case where $\beta^{\prime} \rightarrow-2$ a new type of singularity is attained, in which length-scales tend to zero along the preferred axis of symmetry, but become infinite in spatial directions 
orthogonal to this axis. Such a singularity might be called a "slab" or "infinite pancake". In this case the angle of tilt, $\psi$, is in general non-zero at the singularity. All of these models expand indefinitely for infinitely large proper times. They do not approach isotropy since the ratio of shear to expansion tends to a non-zero limit. Moreover the angle of tilt becomes infinitely large, and in a sense these models only just fail to have Cauchy horizons.

(C) In Fig. 5, the streamlines for $\beta^{\prime}>0$ cross the $\beta^{\prime}$-axis within a finite proper time $t$. One might therefore expect a Cauchy horizon, $H^{+}(S)$, to exist as in Cases (i)-(iv) above. In the spatially homogeneous region, $D_{1}$, matter starts out at a big-bang singularity (where $\mu, \theta, \sigma$, and $\dot{u}$ are infinite) which may be of the cigar, barrel or point type depending on the initial value of $\beta^{\prime}$. Hence the curvature scalar of the homogeneous hypersurfaces may be infinite, finite and non-zero, or zero. As time progresses, the angle of tilt, $\psi$, increases monotonically from its initial value $(\psi=0)$, but as $\psi \rightarrow \infty$ the fluid expansion, shear and acceleration become infinite while the matter density tends to zero. This means that near to $\mathrm{H}^{+}(S)$ the fluid density drops off so rapidly that no fluid crosses the horizon. On the other hand, all quantities associated with the normal congruence remain well-behaved [cf. Cases (i)-(iv) for $1 \leqq \gamma<2$ ] and the fluid lines end in a conformal singularity.

One can continue this spacetime across the horizon by analytic extension along the streamlines in Fig. 5. In the stationary inhomogeneous region, $D_{2}$, the worldlines can be extended for only a finite normal time, $\tilde{t}$, but for an infinite proper time, $t$, and the limiting behaviour is the same as that in the corresponding situation in Case (vA) above $(\theta \rightarrow 0, \mu, \sigma$, and $\dot{u}$ finite and non-zero).

This extension procedure can be expressed in terms of the propagation along a (timelike) curve of initial value data on $\mathrm{H}^{+}(S)$. Imagine a timelike curve which crosses $H^{+}(S)$ at some point $P$, and denote the unit tangent vector to $C$ at $P$ by $X^{a}$. In general the quantity $R_{a b} X^{a} X^{b}$ would be infinite at $P$, but provided one could arrange for the matter density to decay sufficiently rapidly it is possible for $R_{a b} X^{a} X^{b}$ to be well-behaved at $P$. Such a peculiar situation was not considered by Ellis and King [12], because they made the restriction to positive energy density throughout the manifold, $M\{\mu>0$ in $M\}$. The behaviour of the solutions that we have found raises the question of whether or not any results of Ellis and King remain true under the weaker assumption

$$
\{\mu \geqq 0 \text { in } M, \mu>0 \text { on } S\}
$$

where $S$ is some initial (spatially homogeneous) hypersurface. In the next Section we show that the matter density may tend to zero on $\mathrm{H}^{+}(S)$ in other, more general, space-times, and that this peculiar behaviour 
is not a feature which is entirely dependent upon the symmetry of the model, but is more a reflection of the fact that the matter content of the universe is very stiff $(p \approx \mu)$ on $H^{+}(S)$.

\section{Generalizations}

As we have explained in Section 3, the qualitative theory is really only applicable to plane autonomous systems of ordinary differential equations. However, some results pertaining to our analysis of LRS type $V$ models can be generalized. For instance, we now show that in all types of spatially homogeneous models in which a breakdown of prediction can occur, it is possible for the energy density of matter, $\mu$, to be zero on the Cauchy horizon, and non-zero elsewhere. This contrasts sharply with Theorem 4.1 of [12], in which it is shown that under the restriction $\mu>0$ everywhere, Cauchy horizons occur only in a certain subclass of models (Class B), although it is well-known that Cauchy horizons occur in vacuum models which are not of this class (e.g. TaubNUT space $[5,13,14])$.

Theorem 1 (cf. Theorem 4.1 of [12]). There exist tilted homogeneous cosmologies possessing Cauchy horizons for every group type excluding Type I, and for solutions with both zero and non-zero vorticity (i.e. rotation of matter) for every group type excluding Types I and II.

Proof. We need only determine consistent initial data on the null hypersurface $H^{+}(S)$ defined by the relation $\tanh \lambda=1$. The constraint equations (00 and $0 v$; Eq. (2.17) in the normal basis of [10]) must be satisfied, and so must be contracted Bianchi identities (the conservation equation $T_{; b}^{a b}=0$; Eqs. (1.28) and (1.29) of [10]). We shall assume, in common with [12], that the matter content satisfies the reasonable conditions

$$
0 \leqq p \leqq \mu, \quad 0 \leqq \frac{d p}{d \mu} \leqq 1 .
$$

Choosing finite values at time $\tilde{t}=0$ for the expansion tensor, $\tilde{\theta}_{\alpha \beta}$, of the normals to $H^{+}(S)$ and for the geometric quantities $n^{\alpha \beta}$ and $a_{\beta}$ (see Section2), it follows from the constraint equations that $(\mu+p) \sinh \psi \cosh \psi$ and $\mu \cosh ^{2} \psi+p \sinh ^{2} \psi$ are both finite (and possibly zero) on $H^{+}(S)$. With restrictions (5.1) in force, it follows that $\mu=O\left(e^{-2 \psi}\right)$ and $p=O\left(e^{-2 \psi}\right)$ as $\psi \rightarrow+\infty$, i.e. that $\mu \rightarrow 0$ on the horizon. However, we have as yet no guarantee that $\mu \neq 0$ off the horizon. For most reasonable equations of state, $p / \mu$ will approach a well-defined limit as $\mu \rightarrow 0$, and henceforth we shall assume this to to be the case; this means that $\mu e^{2 \psi}$ tends to a finite limit on $\mathrm{H}^{+}(S)$. 
To establish that there exist fluid-filled models, we consider the conservation equations [10]

and

$$
\begin{gathered}
\frac{d \tilde{c}_{\alpha}}{d \tilde{t}}+\varepsilon_{\alpha \gamma \delta} \tilde{c}^{\gamma} \tilde{\Omega}^{\delta}+\tilde{\theta}_{\alpha \beta} \tilde{c}^{\beta}-\tilde{c}_{\alpha}\left(\tilde{c}^{\beta} \tilde{c}^{\gamma} \tilde{\theta}_{\beta \gamma}\right)+\tanh \psi\left[\varepsilon_{\alpha \beta \gamma} \tilde{c}^{\beta} \tilde{c}^{\delta} n_{\delta}^{\gamma}\right. \\
\left.+a_{\alpha}-\tilde{c}_{\alpha} a_{\beta} \tilde{c}^{\beta}\right]=0, \\
\frac{d}{d \tilde{t}} \ln (r \sinh \psi)+\tilde{c}^{\alpha} \tilde{c}^{\beta} \tilde{\theta}_{\alpha \beta}=0,
\end{gathered}
$$

$$
\frac{d}{d \tilde{t}} \ln \left(w \tilde{l}^{3} \cosh \varphi\right)-2 a_{\beta} \tilde{c}^{\beta} \tanh \psi=0,
$$

where $\tilde{c}^{\alpha}$ is the (unit) direction of the projection of the fluid flow vector in the hypersurfaces of homogeneity, $S, \tilde{\theta}_{\alpha \beta}$ is the expansion tensor of the normals $n^{\alpha}$ to $S, \tilde{l} \neq 0$ is an average length defined by $\frac{1}{\tilde{l}} \frac{d \tilde{l}}{d \tilde{t}}=\frac{1}{3} \tilde{\theta}_{\alpha}^{\alpha}=: \frac{1}{3} \tilde{\theta}$, $\tilde{\Omega}^{\alpha}$ is the rate of rotation of the $\left\{\tilde{e}_{\mu}\right\}$ triad along $n^{\beta}, \tilde{t}$ measures proper time along the normals, and $w(w>0)$ and $r(r>0)$ are matter variables defined for $\mu>0$ as in Section 2:

$$
\frac{d w}{w}:=\frac{d \mu}{\mu+p} \text { and } \frac{d r}{r}:=\frac{d p}{d \mu} \cdot \frac{d \mu}{\mu+p} .
$$

Equation (5.2) merely determines the rate of rotation of $\tilde{c}_{\alpha}$ along the normals, whilst Eqs. (5.3) and (5.4) effectively determine the propagation of the energy density, $\mu$, and the angle of tilt, $\psi$.

We next confine the initial data so that

$$
\left(\frac{d p}{d \mu}\right)_{0}\left[\tilde{\theta}-2 a_{\beta} \tilde{c}^{\beta}\right] \neq \tilde{c}^{\alpha} \tilde{c}^{\beta} \tilde{\theta}_{\alpha \beta},
$$

where the suffix 0 refers to the time $\tilde{t}=0$. There is clearly ample freedom to ensure that the restriction (5.5) is satisfied for all group types. Upon eliminating the derivatives of the matter terms in Eqs. (5.3) and (5.4) we find that

$\left(\frac{d p}{d \mu}\right)\left[\tilde{\theta}-2 a_{\beta} \tilde{c}^{\beta} \tanh \psi\right]-\tilde{c}^{\alpha} \tilde{c}^{\beta} \tilde{\theta}_{\alpha \beta}+\left(\frac{d p}{d \mu} \tanh \psi-\operatorname{coth} \psi\right) \frac{d \psi}{d t}=0$

and using restriction (5.5) it follows that $\frac{d \psi}{d \tilde{t}} \neq 0$ when $\tilde{t}=0$. This condition guarantees that the homogeneous hypersurfaces change their

null character off $\tilde{t}=0$.
Now for $\mu \rightarrow 0$ the restrictions $(5.1) \Rightarrow w_{0}\left(\frac{\mu}{\mu_{0}}\right) \leqq w \leqq w_{0}\left(\frac{\mu}{\mu_{0}}\right)^{1 / 2}$, and we have seen that the limit of $\mu e^{2 \psi}$ must be finite. Let us suppose 
first that it is zero. Then $\mu=o\left(e^{-2 \psi}\right) \Rightarrow w=o\left(e^{-\psi}\right)$ as $\tilde{t} \rightarrow 0$. From Eq. (5.4) it follows that $w=o\left(e^{-\psi}\right)$ on $H^{+}(S) \Rightarrow w \equiv 0$ in some neighbourhood of $\tilde{t}=0$. This is possible only in the case $\mu \equiv 0$, which we seek to avoid. Consequently $\mu e^{2 \psi}$ must tend to a non-zero limit and so $\mu \neq 0$ off $H^{+}(S)$.

We have therefore found that it is possible to choose the initial data for a non-empty model whenever one can determine finite $a_{\kappa}, n^{\tau \mu}$ and $\tilde{\theta}_{\alpha \beta}$ such that on $H^{+}(S)$, the restriction (5.5) is satisfied and $\mu e^{2 \psi} \neq o(1)$. This last condition is equivalent to the conditions [10]:

(i) either

$$
3 a_{\kappa} \sigma_{\alpha}^{\kappa}-\varepsilon_{\alpha \kappa \tau} n^{\tau \mu} \tilde{\sigma}_{\mu}^{\kappa} \neq 0 \quad \text { or } \quad \tilde{c}_{\alpha}=0 \quad(\alpha=1,2,3)
$$

and (ii)

$$
-\frac{1}{3} \tilde{\theta}^{2}+\frac{1}{2} \tilde{\sigma}^{\alpha \beta} \tilde{\sigma}_{\alpha \beta}+3 a_{\alpha} a^{\alpha}+\frac{1}{2}\left(n^{\alpha \beta} n_{\alpha \beta}-\frac{1}{2}\left(n_{\gamma}^{\gamma}\right)^{2}\right)+\Lambda \neq 0
$$

where $\tilde{\sigma}_{\alpha \beta}:=\tilde{\theta}_{\alpha \beta}-\frac{1}{3} \tilde{\theta} \delta_{\alpha \beta}$ is the shear tensor. The constraints (5.5) and (5.8) may clearly be satisfied in all models. The only case of a tilted model in which it is impossible to satisfy constraint (5.7) is for Bianchi type $I\left(a_{\kappa} \equiv 0, n^{\tau \mu} \equiv 0\right)$. For such a model $\psi \equiv 0$, and there is no Cauchy horizon.

The vorticity, $\omega$, is non-zero if and only if $n_{\alpha \gamma} n_{\beta}^{\gamma} \tilde{c}^{\alpha} \tilde{c}^{\beta} \neq 0$ [10]. Consequently the only cases of tilted models where this is impossible are for Type $I\left(a_{\kappa} \equiv 0, n^{\tau \mu} \equiv 0\right)$ and Type $I I\left(a_{\kappa} \equiv 0, n_{\alpha \beta} n^{\alpha \beta} \equiv\left(n_{\gamma}^{\gamma}\right)^{2}>0\right)$ (cf. [12]).

One would imagine that if the energy density of matter were ever zero at any finite time, then it might perhaps always be zero. Indeed a theorem on these lines has been proved by Hawking [24], but our result can be reconciled with his when it is recalled that Hawking's theorem is valid in a compact region whose boundary consists of two parts, one on which the energy density vanishes, and the other whose normal is nonspacelike. No such region exists in our case.

It is easy to show that whenever the energy density vanishes in the above manner on the Cauchy horizon, the equation of state of the matter is of the form $p \approx \mu$ in a neighbourhood of the horizon. More precisely, we have

Theorem 2. Suppose that a perfect fluid spatially homogeneous universe model possesses a Cauchy horizon, $H^{+}(S)$, on which the energy density is zero and the commutation functions $\left(a^{\alpha}, n^{\alpha \beta}, \tilde{\theta}^{\alpha \beta}, \tilde{\Omega}^{\alpha}\right)$ in the normal basis are all finite, and that inequalities (5.1) are valid everywhere. Suppose also that $\frac{d p}{d \mu}$ approaches a finite limit as $\mu \rightarrow 0$ (this will be satisfied for most reasonable equations of state). Then $\frac{d p}{d \mu} \rightarrow 1$ and $\frac{p}{\mu} \rightarrow 1$ on $H^{+}(S)$. 
Proof. Let $L:=\lim _{\mu \rightarrow 0} \frac{d p}{d \mu}$. If $L \neq 1$ on $H^{+}(S)$ it follows from Eq. (5.6) that $\frac{d \psi}{d \tilde{t}}$ is bounded as $\tilde{t} \rightarrow 0$, and so $\psi$ does not tend to infinity, a contradiction. Consequently $L=1$, and letting $\mu \rightarrow 0$ with (5.1) holding shows that $\frac{p}{\mu} \rightarrow 1$.

\section{Conclusions: Empty Cauchy Horizons and Structural Instabilities}

We have examined the locally rotationally symmetric models of Bianchi type $V$, and we have depicted their qualitative properties for a variety of equations of state. This has been achieved in particular by employing methods of differential equation theory, and the results are depicted in Figs. 1-5. Table 1 shows the asymptotic values of the important quantities relating to the normal and fluid congruences.

Several interesting results emerge from our analysis, the most surprising of which is the possibility that a universe model, $M$, may consist of two disjoint regions in which the matter density is non-zero, separated by a Cauchy horizon on which the matter density is zero. This result was first obtained by studying a special class of models: axisymmetric generalizations of the open $(k=-1)$ Robertson-Walker solutions. The question then arises as to how dependent this type of behaviour is on the symmetries of the particular model under consideration, and to what extent it is influenced by the particular equation of state of the matter content. We have shown that a wide class of models containing spatially homogeneous regions do indeed possess "empty Cauchy horizons", and there are indications that some inhomogeneous models may have analogous properties. However, it has been shown that whenever the commutation functions are finite near the Cauchy horizon, $H^{+}(S)$, the matter content must mimic that of stiff matter (i.e. $\frac{d p}{d \mu} \rightarrow 1, \frac{p}{\mu} \rightarrow 1$ on $\left.H^{+}(S)\right)$. Thus to obtain such empty Cauchy horizons one must be prepared to admit a very stiff matter content during some stage of the universe's history. For moderate values of the energy density one would expect $0 \leqq p \leqq \frac{1}{3} \mu$ and to this extent one would not consider these empty Cauchy horizons to be realistic ${ }^{5}$.

Another result which is apparent either from our previous discussion or immediately by inspection of Figs. $1-5$ is that the behaviour of the

\footnotetext{
5 Preliminary calculations by King (private communication) indicate that upon relaxing the finiteness condition on the commutation functions, empty Cauchy horizons may exist for a wide variety of equations of state.
} 
Table 1 gives the initial and final states of evolution of the models, for various equations of state. Tabulated are the most relevant quantities: matter density $(\mu)$, curvature scalar of homogeneous hypersurfaces $\left(\tilde{R}^{*}\right)$, fluid acceleration $(\dot{u})$, hyperbolic angles $(\psi, \phi)$, fluid and normal proper time $(t, \tilde{t})$, fluid and normal expansion $(\theta, \tilde{\theta})$, fluid and normal shear $(\sigma, \tilde{\sigma})$, and length-scales in the rest-spaces of observers moving along the fluid and normals $(l, \tilde{l})$. The normal acceleration and the fluid and normal rotation (vorticity) are all zero. Entries are listed as $0, f$, and $\infty$, corresponding respectively to zero, finite and non-zero, and infinite limiting values (real or complex: in the inhomogeneous region some, quantities, e.g. $\tilde{\theta}$, are imaginary). Models are described with the initial state (in the sense of evolution in Figs. 1-5) in the first line, and the final state in the last. An intermediate stage (the horizon) is described for whimper models

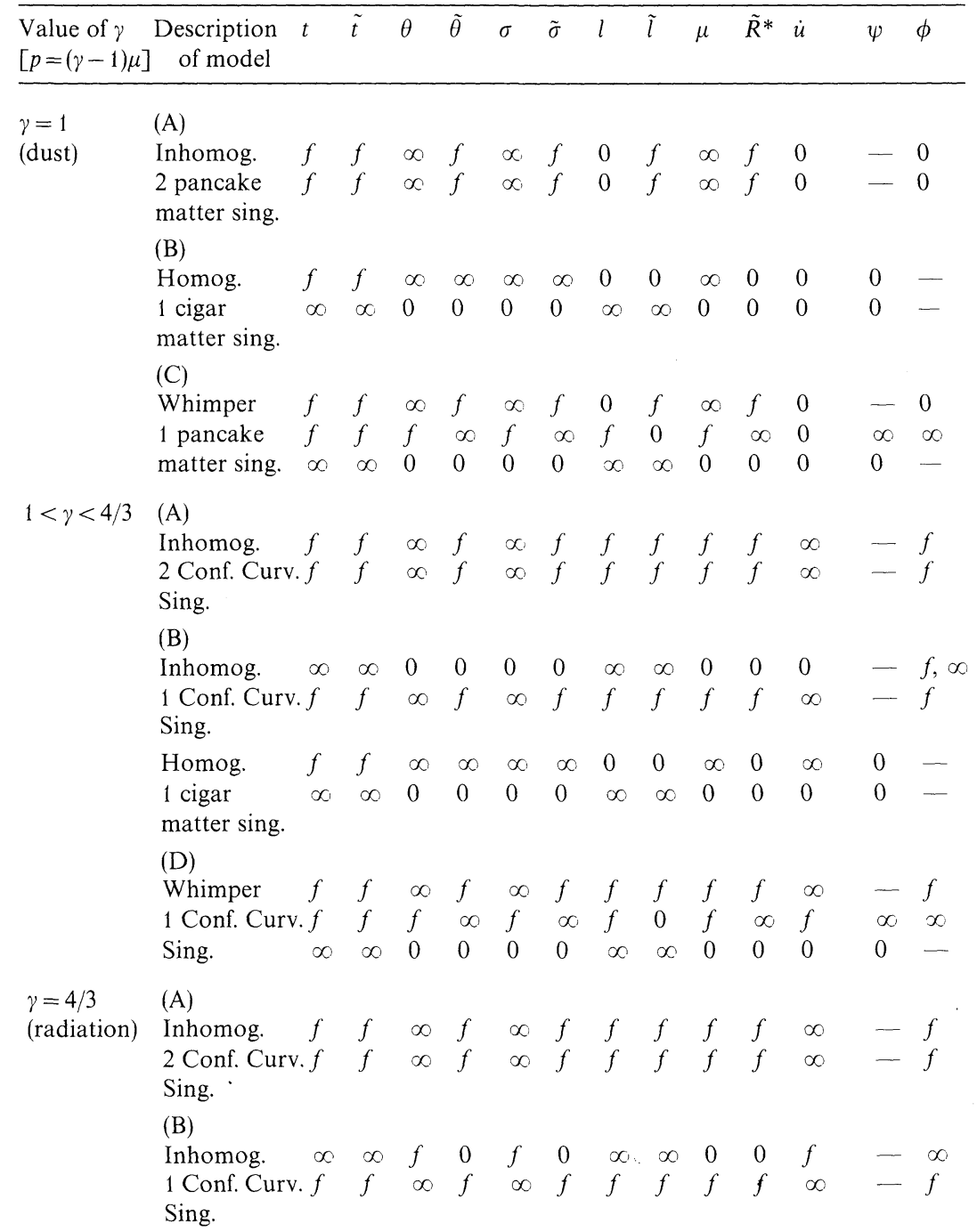


Table 1 (continued)

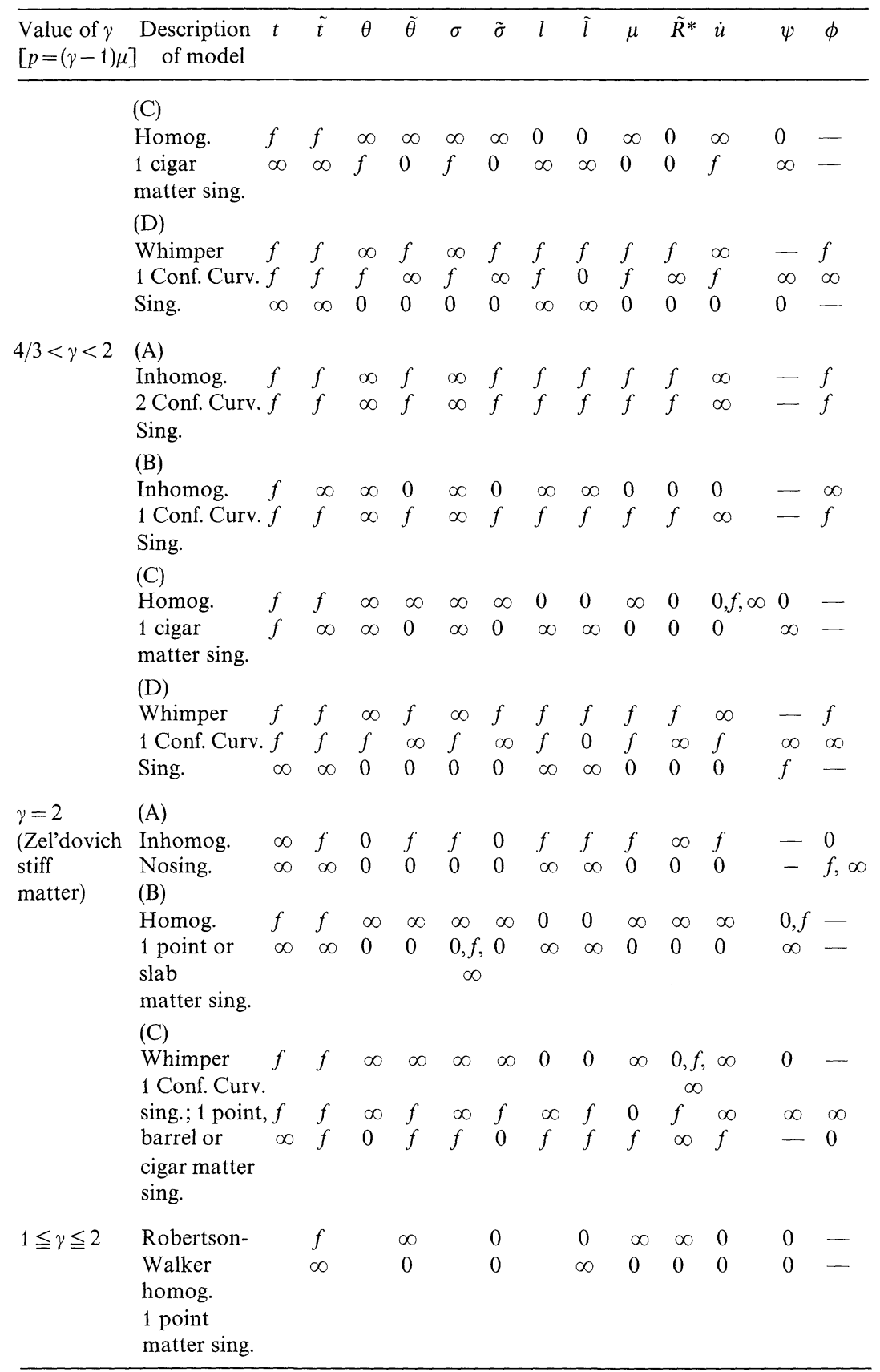


models under consideration varies dramatically with the equation of state: the field equations are structurally unstable against changes in the matter content (cf. [17]). This is a rather disturbing feature of our models, because one might hope that in a realistic model of the universe, any broad properties would be stable against small changes in the matter content. Particularly disquieting is the fact that there is evident structural instability of the dust, radiation and Zel'dovich stiff matter universe models. From an examination of the conservation equations, it seems likely that this property is held in common with more general types of tilted models, and one is therefore led to wonder if such structural instabilities are very special, or if they really do apply on a wider scale to other considerations in cosmology. How well, for instance, do dust $(p=0)$ models of the universe approximate to the late stages of the real universe $(p \approx 0)$ ? Are the radiation models $\left(p=\frac{1}{3} \mu\right)$ stable approximations to the early stages of the universe $\left(p \approx \frac{1}{3} \mu\right)$ ? The answers to such questions would seem to play a crucial role not only in our understanding of the idiosyncracies of Einstein's field equations, but also in our perception of the evolution of the universe.

Acknowledgements. I am grateful to G. F. R. Ellis and A. R. King for communicating details of their work before publication, and for several enlightening discussions on certain aspects of my results. This work was carried out while I held research fellowships with the Science Research Council and at Trinity College, Oxford.

\section{References}

1. Hawking, S. W., Penrose, R.: Proc. Roy. Soc. A 314, 529 (1970)

2. Hawking, S. W., Ellis, G.F. R.: Astrophys. J. 152, 25 (1968)

3. Hawking, S. W., Sciama, D. W.: Comments Astrophys. Space Phys. 1, 1 (1969)

4. Gödel, K.: Rev. Mod. Phys. 21, 447 (1949)

5. Taub, A. H.: Ann. Math. 53, 472 (1951)

6. Schücking, E., Heckmann, O.: World models. In: Stoops, R. (Ed.): Institut International de Physique Solvay, Onzième Conseil de Physique, 1958, p. 149, Belgium, 1959; Heckmann, O., Schücking, E.: Relativistic cosmology. In: Witten, L. (Ed.): Gravitation: an introduction to current research, p. 438. New York: John Wiley and Sons 1962

7. Estabrook,F.B., Wahlquist,H.D., Behr,C.G.: J. Math. Phys. 9, 497 (1968); Ellis, G.F.R., MacCallum, M.A.H.: Commun. math. Phys. 12, 108 (1969)

8. MacCallum, M.A.H.: Commun. math. Phys. 20, 57 (1971); Hawking, S. W.: Monthly Notices Roy. Astron. Soc. 142, 129 (1969); Misner, C. W.: Astrophys. J. 151, 431 (1968), Phys. Rev. Letters 22, 1071 (1969)

9. Collins, C. B., Hawking, S. W.: Astrophys. J. 180, 317 (1973)

10. King, A. R., Ellis, G. F. R.: Commun. math. Phys. 31, 209 (1973)

11. Shepley, L. C.: Phys. Lett. 28 A, 695 (1969)

12. Ellis, G.F.R., King, A.R.: Was the big-bang a whimper? Cambridge University, Preprint (1973)

13. Newman,E.T., Tamburino, L., Unti, T.: J. Math. Phys. 4, 915 (1963) 
14. Misner, C. W.: J. Math. Phys. 4, 924 (1963); Taub-NUT Space as a Counterexample to almost anything. In: Ehlers, J. (Ed.): Relativity theory and astrophysics. I. Relativity and cosmology. Lectures in Applied Mathematics, 8, 160; Am. Math. Soc., Providence, Rhode Island (1967); Misner,C.W., Taub,A.H.: Zh. Eksperim. Teor. Fiz. 55, 233 (1968), translated in Soviet Physics JETP 28, 122 (1969)

15. Hawking, S.W., Ellis, G.F.R.: The large scale structure of space-time. Cambridge University Press (1973)

16. MacCallum, M. A.H., Taub, A.H.: Commun. math. Phys. 25, 173 (1972); Ryan, M.P.: Hamiltonian cosmology. Lecture Notes in Physics, 13. Berlin-Heidelberg-New York: Springer 1972

17. Collins, C. B.: Commun. math. Phys. 23, 137 (1971); 27, 37 (1972); Shikin, I.S.: Zh. Eksperim. Teor. Fiz. 63, 1529 (1972), translated in Soviet Physics JETP, 36, 811 (1973)

18. Farnsworth, D. L.: J. Math. Phys. 8, 2315 (1967)

19. Shikin, I. S.: Commun. math. Phys. 26, 24 (1972)

20. Ellis, G. F. R.: J. Math. Phys. 8, 1171 (1967); Stewart, J. M., Ellis, G. F. R.: J. Math. Phys. 9, 1072 (1968)

21. Nemytskii,V.V., Stepanov,V.V.: Qualitative theory of differential equations. Princeton University Press; Sansone, G., Conti, R.: Non-Linear Differential Equations, Oxford: Pergamon Press 1964; Vojtasek, S., Janac, K.: Solution of non-linear systems. London: Iliffe Books Ltd. (1969)

22. Bendixson, I.: Acta math. 24, 1 (1901)

23. Thorne, K.S.: Astrophys. J. 148, 51 (1967)

24. Hawking, S. W.: Commun. math. Phys. 18, 301 (1970)

Communicated by J. Ehlers

C. B. Collins

University of Cambridge

Institute of Astronomy

The Observatories

Madingley Road

Cambridge CB3 OHA, England 
